\title{
Experimental and Model Investigation of the Oscillatory Transenantiomerization of $L-\alpha$-Phenylalanine
}

\author{
Mieczysław Sajewicz, ${ }^{1}$ Monika Gontarska, ${ }^{1}$ \\ Lukasz Wojtal, ${ }^{1}$ Dorota Kronenbach, ${ }^{1}$ Marcin \\ Leda, ${ }^{2}$ Irving R. Epstein, ${ }^{2}$ and Teresa Kowalska ${ }^{1}$ \\ ${ }^{1}$ Institute of Chemistry, University of Silesia, Katowice, Poland \\ ${ }^{2}$ Chemistry Department, Brandeis University, \\ Waltham, MA, USA
}

\begin{abstract}
In an earlier study, we obtained experimental evidence of the oscillatory transenantiomerization of selected profen drugs (e.g., $S$ - $(+)$ ibuprofen, $S$-(+)-naproxen, and $S$-(+) and $R$-(-)-flurbiprofen) dissolved in aqueous, aqueous-organic, and purely organic liquid media. This process was apparently catalyzed by basic or amphiprotic environments and involved ketoenol tautomerism, and the self-organization of molecules in the solution via association of the carboxylic functional group of profens through hydrogen bonding to form mixed $\mathrm{H}$-bonded associates with the remaining constituents of the solution. A model of the oscillatory transenantiomerization of profens was also developed by adapting an earlier oscillatory model, the Templator. Our new model comprises two linked Templators. The essence of the Templator model adapted to the oscillatory transenantiomerization of profens is the assumption that the H-bonded profen homodimer acts as a template, able to generate new dimers having the same steric configuration as their respective monomeric units.

As profens belong to the class of 2-arylpropionic acids (2-APAs), we concluded that the phenomenon of oscillatory transenantiomerization may occur in other 2-APAs as well, among them those amino acids whose molecular structure can formally be derived from propionic acid. Thus, in this study, we
\end{abstract}

Address correspondence to Teresa Kowalska, Institute of Chemistry, University of Silesia, 9 Szkolna Street, 40-006 Katowice, Poland. E-mail: kowalska@us.edu.pl 
focus our attention on $L$ - $\alpha$-phenylalanine (LPA; one of the nine amino acids essential for humans). Using thin layer chromatography (TLC) and polarimetry, we demonstrate the ability of LPA to undergo oscillatory transenantiomerization analogous to that observed with profens. The selforganization of molecules in a $70 \%$ ethanol solution of LPA is confirmed with photographs taken in UV light $(\lambda=254 \mathrm{~nm})$. Finally, we propose a skeleton molecular mechanism for the transenantiomerization of LPA and simulate the oscillatory interconversion of its $L$ and $D$ forms with two linked Templators.

Keywords: Keto-enol tautomerism, $L$ - $\alpha$-phenylalanine, LPA, Oscillatory transenantiomerization, Polarimetry, Templator, TLC

\section{INTRODUCTION}

In our earlier studies, we have reported on the oscillatory transenantiomerization of selected profen drugs in aqueous, aqueousorganic, and purely organic solvents. ${ }^{[1-3]}$ We proposed a mechanism for their structural conversion via keto-enol tautomerism, and we provided experimental evidence of the catalytic role of basic environments in this conversion. ${ }^{[4]}$ In chemical terms, profen drugs belong to the class of 2-arylpropionic acid (2-APAs), and their chirality center is located on the $\alpha-C$ atom of the acid molecule. Schematically, structural conversion of profens is shown in the following scheme:

$$
S \text {-(+)-profen } \leftrightarrow \text { keto-enol tautomer } \leftrightarrow R \text {-(-)-profen }
$$

In addition to profens, many other compounds belong to the class of 2 -arylpropionic acids, among them those $\alpha$-amino acids whose structure can, formally at least, be derived from propionic acid. Thus, in the first instance, we focused our attention on $L$ - $\alpha$-phenylalanine (LPA), one of the nine amino acids essential for humans. We were curious to know whether $L$ - $\alpha$-phenylalanine can, when dissolved in certain solvents, undergo an analogous oscillatory transenantiomerization to $D$ - $\alpha$-phenylalanine (DPA), as is the case with profens. In other words, we wanted to establish whether reaction (2) can take place in aqueousorganic solutions of $L-\alpha$-phenylalanine in an oscillatory manner:

$$
\text { LPA } \leftrightarrow \text { keto-enol tautomer } \leftrightarrow \text { DPA }
$$

The results obtained in our study demonstrate that $L$ - $\alpha$-phenylalanine dissolved in low molecular weight solvents undergoes structural conversion to its $D$ form with an ease similar to that of the earlier investigated profens. 


\section{EXPERIMENTAL}

\section{$L-\alpha$-Phenylalanine}

The chemical structure of $L$ - $\alpha$-phenylalanine is given in Fig. 1. Its isoelectric point $\mathrm{p} I$ is 5.48. In our study, we used $L$ - $\alpha$-phenylalanine purchased from Merck KGaA (Darmstadt, Germany; cat. \# 1.07256.0025). In the thin-layer chromatographic experiments, we used solutions of $L-\alpha$-phenylalanine in ethanol-water (7:3, v/v), ethanol-basic buffer, $\mathrm{pH}=9(7: 3, \mathrm{v} / \mathrm{v})$, and ethanol-glacial acetic acid $(7: 3, \mathrm{v} / \mathrm{v})$, its concentration always being equal to $1 \mathrm{mg} \mathrm{mL}^{-1}$ (ca. $6.1 \times 10^{-3} \mathrm{~mol} \mathrm{~L}^{-1}$ ). The $L$ - $\alpha$-phenylalanine solutions were stored for 8 days at $22 \pm 2^{\circ} \mathrm{C}$ in order to trace the oscillatory transenantiomerization. At certain time intervals, the solution samples were analyzed by TLC.

In the polarimetric experiment, we used the analogous three types of $L-\alpha$-phenylalanine solutions, with the amino acid concentration always equal to $5 \mathrm{mg} \mathrm{mL}^{-1}$ (ca. $3.03 \times 10^{-2} \mathrm{~mol} \mathrm{~L}^{-1)}$.

\section{Polarimetric Measurements of the Specific Rotation $[\alpha]_{D}$}

Measurements of the specific rotation $\left([\alpha]_{D}\right)$ of $L$ - $\alpha$-phenylalanine solutions in ethanol-water, ethanol-basic buffer, and ethanol-glacial acetic acid mixtures were carried out at $9 \pm 2^{\circ} \mathrm{C}$ and $22 \pm 2^{\circ} \mathrm{C}$ for $240 \mathrm{~min}$ (in 10-min intervals), with and without strirring (by ultrasonication of the measuring cell). Ultrasonication was performed with a model RK 255H Sonorex Super (Bandelin, Berlin, Germany) ultrasonication bath. Measurements of the specific rotation were carried out with a Polamat

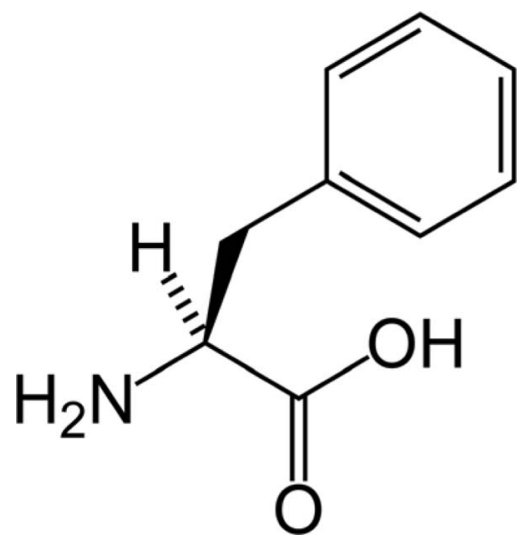

Figure 1. Chemical structure of $L-\alpha$-phenylalanine. 
A model polarimeter (manufactured by Carl Zeiss, Jena, Germany). The optical path length of the measuring cell was exactly $10 \mathrm{~cm}(=1 \mathrm{dm})$, and its volume was ca. $1 \mathrm{~mL}$. The specific rotation $[\alpha]_{D}$ was calculated as

$$
[\alpha]_{D}=100 \alpha / c d
$$

where $\alpha$ is the measured rotation (in degrees); $D$ is the wavelength employed, $\lambda=589 \mathrm{~nm}$, which corresponds to the sodium $D$ line; $\mathrm{c}$ is the concentration of a given compound in $\mathrm{g}(100 \mathrm{~mL})^{-1}$ solution; and $\mathrm{d}$ is the sample thickness in $\mathrm{dm}$.

From the literature ${ }^{[5]}$ it is known that specific rotation $\left([\alpha]_{D}\right)$ of $L-\alpha$-phenylalanine at $20^{\circ} \mathrm{C}$ is ca. $-34^{\circ}$.

\section{Commercial TLC Silica Gel Layers and Their Pretreatment}

TLC was performed on commercial glass plates $(20 \mathrm{~cm} \times 20 \mathrm{~cm})$ precoated with $0.25 \mathrm{~mm}$ layers of silica gel $60 \mathrm{~F}_{254}$ (Merck KGaA, Darmstadt, Germany; cat. \#1.05715). Before use, the plates were carefully washed by predevelopment with methanol-water, 9:1 (v/v), and then dried at ambient temperature $\left(22 \pm 2^{\circ} \mathrm{C}\right)$ for $3 \mathrm{~h}$.

The washed and dried plates were then twice impregnated by conventional dipping for $2 \mathrm{~s}$. The first solution used for impregnation was a $2.39 \times 10^{-2} \mathrm{M}$ aqueous solution of $\mathrm{CuSO}_{4}$ (analytical grade; POCh, Gliwice, Poland). After the first impregnation, the plates were dried for $10 \mathrm{~min}$ at $105-110^{\circ} \mathrm{C}$ in a thermostatted chamber. The second solution used for impregnation was $3.04 \times 10^{-2} \mathrm{M} L$-proline ( $L$-Pro; Merck; cat. $\# 1.07434 .0010)$ in a water-methanol mixture $(9: 1, \mathrm{v} / \mathrm{v})$. It is noteworthy that the molar ratio of $\mathrm{Cu}^{2+}$ cations to $L$-Pro molecules deposited on the chromatographic plates was 1:2, allowing the in situ formation of the $\left[\mathrm{Cu}(L-\mathrm{Pro})_{2}\right]^{2+}$ complex. After the second impregnation, the plates were dried again at ambient temperature for $3 \mathrm{~h}$. Finally, the impregnated adsorbent layers were ready for chromatography.

\section{Mobile Phase and Development of Thin Layer Chromatograms}

Development of the stored $L-\alpha$-phenylalanine (LPA) samples was carried out at $22 \pm 2{ }^{\circ} \mathrm{C}$ for a distance of $15 \mathrm{~cm}$ in the one-dimensional mode using a ternary mobile phase composed of n-butanol (n-BuOH)acetonitrile $(\mathrm{ACN})$-water $\left(\mathrm{H}_{2} \mathrm{O}\right)$ in the proportion 6:2:3 (v/v). The anticipated mechanism of retention with each of the two $\alpha$-phenylalanine (PA) enantiomers LPA and DPA is given below:

$$
\begin{aligned}
& {\left[\mathrm{Cu}(L \text {-Pro })_{2}\right]^{2+}+\mathrm{LPA} \leftrightarrow[\mathrm{Cu}(L \text {-Pro })(\mathrm{LPA})]^{2+}+L \text {-Pro; } K_{1}} \\
& {\left[\mathrm{Cu}(L-\mathrm{PrO})_{2}\right]^{2+}+\mathrm{DPA} \leftrightarrow[\mathrm{Cu}(L \text {-Pro })(\mathrm{DPA})]^{2+}+L \text {-Pro; } K_{2}}
\end{aligned}
$$


The above mechanism of enantioseparation is known as ligand exchange chromatography (or complexation chromatography). The TLC procedure employed in this study was adapted from that developed by Bhushan et al. ${ }^{[6]}$ to use commercially precoated chromatographic glass plates in place of the laboratory-coated ones used earlier.

Sample application to the plates was performed with an autosampler (AS 30, Desaga, Heidelberg, Germany). The $\alpha$-phenylalanine solutions were applied to the plate $1.5 \mathrm{~cm}$ above the lower edge of the plate in aliquots of $5 \mu \mathrm{L}$ spot $^{-1}$. Nine samples at equal distances of $2 \mathrm{~cm}$ from one another were applied per plate, and then the chromatogram was developed in the one-dimensional ascending mode. After development, the plates were dried at ambient temperature for $3 \mathrm{~h}$, and each development track was densitometrically scanned in 1-mm intervals at a width of $1 \mathrm{~cm}$ in the direction of development. Each experiment was carried out on two plates; thus each numerical result given in this paper is a mean obtained from 18 individual development lanes.

\section{Densitometric Assessment of the Thin Layer Chromatograms}

Densitograms were acquired with a Desaga (Heidelberg, Germany) Model CD 60 densitometer equipped with Windows-compatible ProQuant software. Concentration profiles of the development lanes for $\alpha$-phenylalanine were recorded in ultraviolet (UV) light from a deuterium lamp (in the reflectance mode) at $200 \mathrm{~nm}$. The dimensions of the rectangular light beam were $2.0 \mathrm{~mm} \times 0.1 \mathrm{~mm}$. The maxima of the concentration profiles were used for calculation of $R_{\mathrm{F}}$ values.

\section{Photography}

A colorless, apparently homogeneous, and perfectly transparent solution of $L$ - $\alpha$-phenylalanine in the ethanol-water mixture, $7: 3, v / v(3.03 \times$ $10^{-2} \mathrm{~mol} \mathrm{~L}^{-1}$ ) was poured onto a watchglass, illuminated in the dark chamber used for visualization of the thin layer chromatograms with UV light $(\lambda=254 \mathrm{~nm})$, and photographed with an amateur digital camera to reveal the lack of homogeneity, imperceptible in daylight, of the $L-\alpha$-phenylalanine solution.

\section{RESULTS AND DISCUSSION}

\section{Thin Layer Chromatography}

One of the most efficient separation techniques, chromatography can provide direct proof of structural conversion of a given enantiomer to its 
mirror image, as it can enantioseparate the two species and in that way demonstrate their coexistence. TLC, with its simplicity and robustness, has in its arsenal a number of effective methods of enantioseparating amino acids, including $L, D$ - $\alpha$-phenylalanine. In this study, we benefited from the TLC method elaborated by Bhushan et al. ${ }^{[6]}$ and adapted here to employ commercial chromatographic plates.

The aim of our TLC experiments was to gather evidence of the occurence of the oscillatory transenantiomerization of $L-\alpha$-phenylalanine to its $D$-form, with $L$ - $\alpha$-phenylalanine samples dissolved in the three different mixed solvents (i.e., ethanol-water, ethanol-basic buffer, $\mathrm{pH}=9$, and ethanol-glacial acetic acid, 7:3 (v/v)), and then stored for eight days at $22 \pm 2{ }^{\circ} \mathrm{C}$. As in our earlier studies on the oscillatory transenantiomerization of selected profens, described in papers, ${ }^{[1-3]}$ the $L$ - $\alpha$-phenylalanine concentration in the freshly made solutions was kept low (ca. $6.1 \times 10^{-3} \mathrm{~mol} \mathrm{~L}^{-1}$ ) in order to facilitate enantioseparation and to obtain well-defined Gaussian concentration profiles of the separated analytes.

In Fig. 2, we show the oscillatory changes of the retardation factor $\left(R_{\mathrm{F}}\right)$ with the three different $L$ - $\alpha$-phenylalanine solutions stored at $22 \pm 2{ }^{\circ} \mathrm{C}$ for eight days. These oscillatory changes demonstrate the structural conversion of $L$ - $\alpha$-phenylalanine to the $D$ antimer, as the amino acid does not decompose under the storage conditions employed and the experimental error of the retardation factor $\left(R_{\mathrm{F}}\right)$ measurements

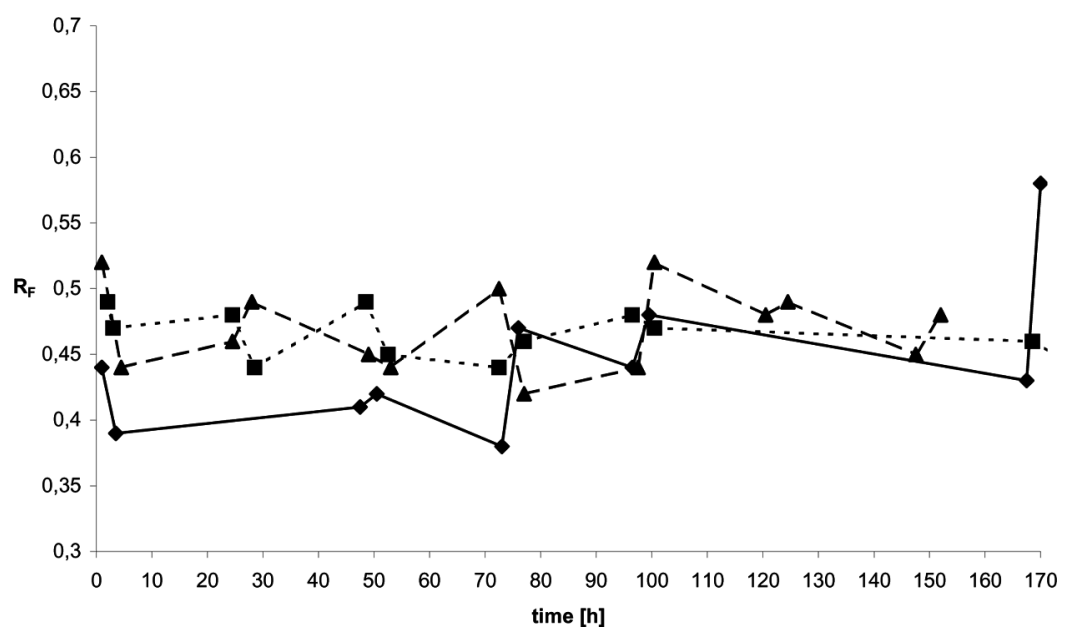

Figure 2. Dependence of retention, $R_{\mathrm{F}}$, for $L$ - $\alpha$-phenylalanine dissolved in $(\boldsymbol{\nabla})$ ethanol - water, 7:3 (v/v); (ם) ethanol - basic buffer, $\mathrm{pH}=9,7: 3(\mathrm{v} / \mathrm{v})$; and (ム) ethanol - glacial acetic acid, 7:3(v/v), on sample storage time $\left(R_{\mathrm{F}}=f(t)\right)$ at $22 \pm 2{ }^{\circ} \mathrm{C}$. 
never exceeds $\pm 0.02 R_{\mathrm{F}}$ units. The $R_{\mathrm{F}}$ values of the peak maxima for the analyte dissolved in the ethanol-aqueous medium oscillated between $0.58 \pm 0.02$ and $0.37 \pm 0.02$. For the analyte dissolved in the ethanolbasic buffer, the $R_{\mathrm{F}}$ values of the peak maxima oscillated between $0.49 \pm 0.02$ and $0.42 \pm 0.02$, and for the same analyte dissolved in the ethanol-glacial acetic acid, the analogous values oscillated between $0.52 \pm 0.02$ and $0.42 \pm 0.02$. As can be seen from the three plots shown in Fig. 2, in each stored solution the oscillations of the $R_{\mathrm{F}}$ value significantly surpass the measuring error.

For the sake of comparison, we recall the analogous results of Bhushan et al., ${ }^{[6]}$ in which the chromatographic system (in a variation of the version employed in this study) was first introduced. The $R_{\mathrm{F}}$ value reported there for $L$ - $\alpha$-phenylalanine was 0.39 and that for the $D$ antimer was 0.32 . The $R_{\mathrm{F}}$ values obtained in our modified chromatographic system are somewhat higher than in the original system, and the peak resolution in the original system $\left(\Delta R_{\mathrm{F}}=0.07\right)$ was less than the difference between the extremal peak positions observed in the modified system $\left(\Delta R_{\mathrm{F}}\right.$ up to 0.21 , as observed for $\alpha$-phenylalanine stored in the ethanol-aqueous medium).

The highest average level of $R_{\mathrm{F}}$ is observed for the ethanol-glacial acetic acid mixture, and the lowest is found in the ethanol-water mixture. The TLC results confirm the role played by acidic and basic environments in the process of transenantiomerization. Namely, from earlier studies, ${ }^{[6]}$ it is known that, in this chromatographic system, the $R_{\mathrm{F}}$ values for $L-\alpha$-phenylalanine are higher than those for its $D$ antimer. The higher average $R_{\mathrm{F}}$ values observed for $L$ - $\alpha$-phenylalanine stored in ethanol-glacial acetic acid imply that the degree of steric conversion in this solvent is lower than in ethanol-basic buffer or in ethanol-water (i.e., glacial acetic acid hampers transenantiomerization, whereas basic and amphiprotic environments exert a catalytic effect).

A simultaneous demonstration of the changing maximum positions and shapes of the concentration profiles for the sample stored in the amphiprotic ethanol-water mixture is presented as a sequence of snapshots in Figs. 3(a)-(f). For the ethanol-basic buffer and the ethanolglacial acetic acid, the changing peak positions are analogous to the ethanol - water case. However, only in the ethanol-water solvent did we obtain an occasional (and partial) enantioseparation of the two amino acid antimers.

Why do we consider the changing positions of the analyte's maximum $R_{\mathrm{F}}$ values and the changing shapes of its concentration profile as the most convincing demonstration of the oscillatory transenantiomerization of 2-APAs? Because our experience suggests that the oscillatory structural conversion of amino acid (and also profen) 

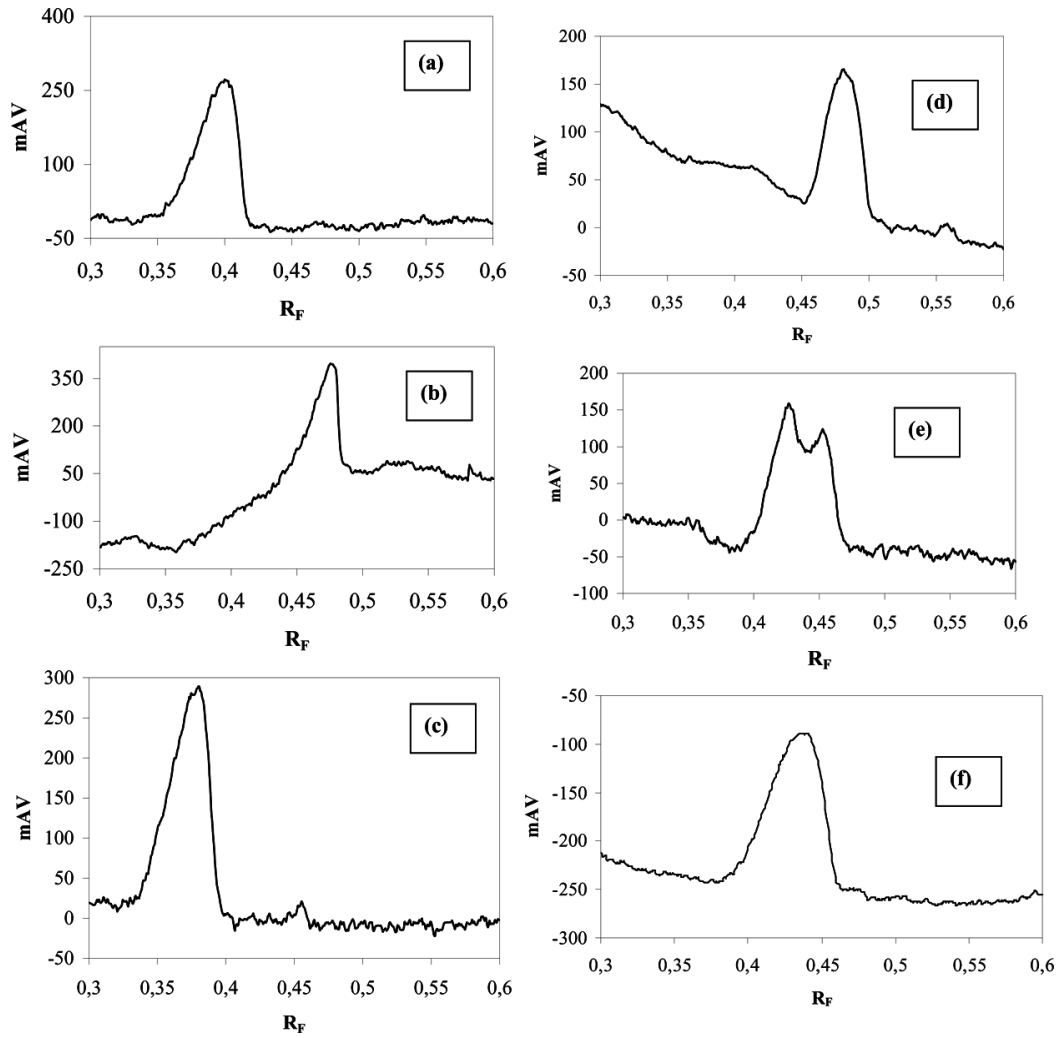

Figure 3. Sequence of densitometric concentration profiles for the $L-\alpha$-phenylalanine solution in ethanol - water, 7:3 (v/v) after (a) $47.5 \mathrm{~h}$; (b) $50.5 \mathrm{~h}$; (c) $73 \mathrm{~h}$; (d) $96.5 \mathrm{~h}$; (e) $99.5 \mathrm{~h}$; and (f) $167.5 \mathrm{~h}$ storage time at $22 \pm 2{ }^{\circ} \mathrm{C}$.

molecules is very rapid and, in most cases, we are not able to chromatographically catch the exact moment at which the two antimers appear in the solution side by side as the racemic mixture. Far more often, the chromatogram shows either of the two antimers in a pure, or in a strongly predominant form. We also managed to demonstrate that the chromatographic process - and specifically, the spontaneous stirring of the migrating sample which passes through a labyrinth of randomly arranged microchannels - can significantly affect the transenantiomerization and, in extreme cases, it can even result in nearly full racemization of the optically pure starting material, as was the case with $S$-(+)- and $R$-(-)-flurbiprofen. ${ }^{[9]}$

Sometimes, however, a rare case can be captured when the two antimers derived from a single optically pure enantiomer appear chromatographically separated in comparable proportions. 


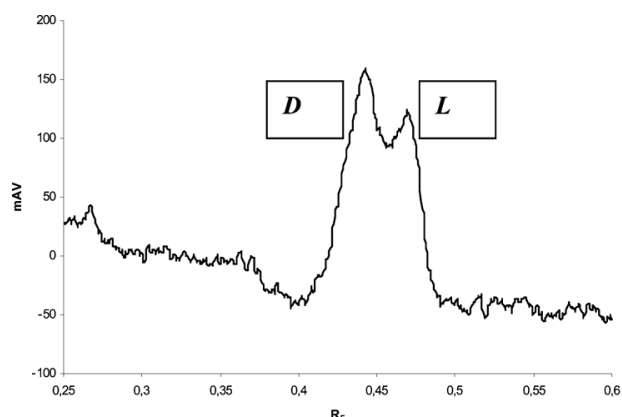

(a)

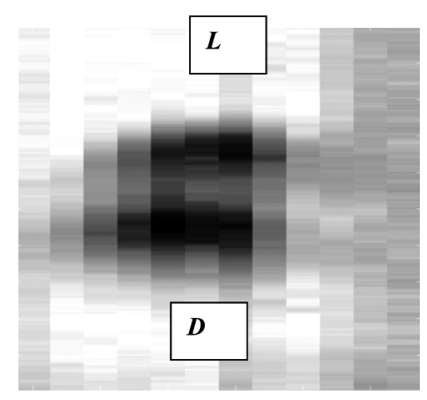

(b)

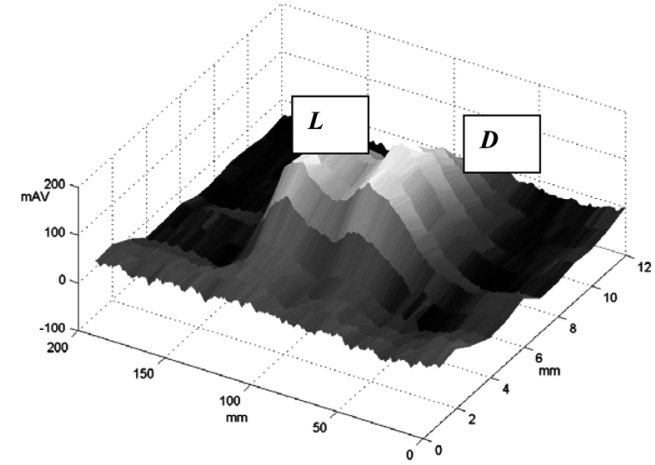

(c)

Figure 4. 1D (densitogram) (a), 2D (b) and 3D (c) representations of the two enantioseparated chromatographic spots of $L$ - $\alpha$-and $D$ - $\alpha$-phenylalanine after sample storage for $99.5 \mathrm{~h}$ in ethanol - water solution $(7: 3, \mathrm{v} / \mathrm{v})$ at $22 \pm 2{ }^{\circ} \mathrm{C}$. The $2 \mathrm{D}$ and $3 \mathrm{D}$ pictures were drawn on the densitometric scans of the separated pair of $L, D$ - $\alpha$-phenylalanine antimers taken at 1 -mm intervals.

In Figs. 4(a)-(c), we give a typical example of an incomplete enantioseparation of $L$ - $\alpha$-phenylalanine from its $D$ antimer for a sample stored for a longer period of time $(99.5 \mathrm{~h})$ in the amphiprotic ethanol-water solution.

\section{Photography}

Chemical oscillatory processes often follow a kinetic-diffusive mechanism. ${ }^{[7]}$ The diffusive effect may arise from the density anisotropy of the reaction medium, which can be caused by several factors. Among these factors are: the gelating property of a constituent of the reaction medium; liquid-crystalline organization of the molecules of the reaction mixture; formation of an intermediate or product faster than its diffusion in the reaction medium, etc. 
In the case of the oscillatory transenantiomerization of profens, first described in papers, ${ }^{[1-3]}$ it is well documented that these compounds possess a gelating property and/or an ability to organize the low molecular weight molecules of the respective solvents in such a way as to result in the density anisotropy of the liquid reaction mixture. In a paper ${ }^{[8]}$ we provided abundant experimental evidence (in the form of viscosimetric, high performance liquid chromatographic (HPLC), and ${ }^{1} \mathrm{HNMR}$ spectroscopic data) pointing to the lack of homogeneity of profen solutions.

Here, we document the density anisotropy of the colorless, trasparent and, in daylight, apparently homogenous solution of $L-\alpha$-phenylalanine in $70 \%$ aqueous ethanol in the most direct way, namely, by photographing this solution in UV light at $254 \mathrm{~nm}$ (Fig. 5). The lack of density homogeneity of this $L$ - $\alpha$-phenylalanine solution poured onto a watchglass is evident from the regular series of concentric stripes that make a jelly-like impression. This result strongly supports the notion that the oscillatory structural conversion of $L-\alpha$-phenylalanine can follow a kinetic-diffusion mechanism.

\section{Polarimetry}

The specific rotation of $L-\alpha$-phenylalanine solutions in ethanol-water, ethanol-basic buffer, and ethanol-glacial acetic acid binary mixtures

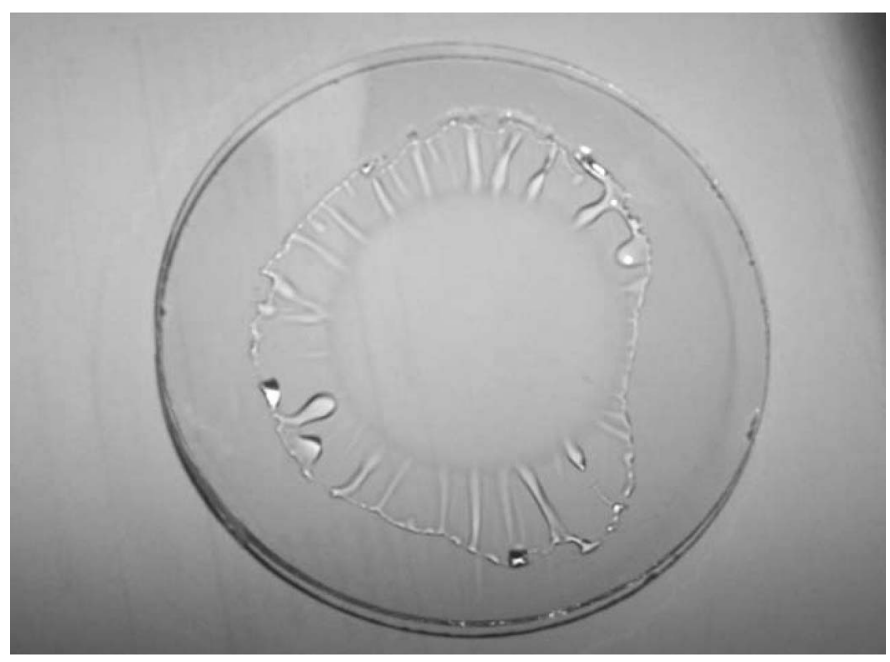

Figure 5. Photograph of a $70 \%$ aqueous ethanol solution of $L$ - $\alpha$-phenylalanine on a watchglass, taken in UV light at $254 \mathrm{~nm}$. 
(with the $L$ amino acid concentration always equal to $3.03 \times 10^{-2} \mathrm{~mol}$ $\mathrm{L}^{-1}$ ) was measured for $240 \mathrm{~min}$ by polarimetry at two different working temperatures, $9 \pm 2{ }^{\circ} \mathrm{C}$ and $22 \pm 2^{\circ} \mathrm{C}$. At each temperature, one sample was kept unstirred and the other was stirred by ultrasonication, in order to also investigate the effect of stirring.

At both working temperatures, with and without stirring, the specific rotation of the $L-\alpha$-phenylalanine solutions was not constant, but underwent oscillatory changes. The amplitude of these oscillations at $22 \pm 2^{\circ} \mathrm{C}$ was considerably lower than at $9 \pm 2{ }^{\circ} \mathrm{C}$. This effect can probably be ascribed to the lower viscosity of the samples kept at $22 \pm 2^{\circ} \mathrm{C}$ and, hence, to the less pronounced density anisotropy of these solutions. The results obtained at $9 \pm 2^{\circ} \mathrm{C}$ are shown in Figs. 6(a)-(c).

From the results in Figs. 6(a)-(c), the following conclusions can be drawn. First, the amplitude of oscillation in the stirred samples is, in most cases, lower than in the unstirred ones. We conclude that stirring the solutions tends to destroy their density anisotropy and, hence, to hamper the oscillatory transenantiomerization of $L-\alpha$-phenylalanine. Secondly, the highest amplitude of oscillations is observed in the basic environment [Fig. 6(b)], somewhat lower in the amphiprotic one [Fig. 6(a)], and the lowest in the acidic environment [Fig. 6(c)]. These results coincide well with our earlier findings ${ }^{[4]}$ as to the catalytic influence of basic and amphiprotic environments on the oscillatory steric conversion of the chiral 2-arylpropionic acids via the keto-enol tautomerism. Finally, the polarimetric results combined with the TLC data provide strong evidence in favor of oscillatory transenantiomerization of $L$ - $\alpha$-phenylalanine dissolved in low molecular weight solvents and stored for long periods of time.

\section{Modeling of the Oscillatory Transenantiomerization of $L$ - $\alpha$-Phenylalanine}

Oscillatory chemical reactions are relatively rare, and elucidating the molecular mechanism of such processes is a considerable challenge. We have proposed a preliminary model of the oscillatory transenantiomerization of profens and of $L-\alpha$-phenylalanine. ${ }^{[10,11]}$ Here, we introduce the model, applied to the oscillatory transenantiomerization of $L-\alpha$-phenylalanine, in somewhat greater detail, considering spatial as well as temporal behavior.

The stoichiometry of the oscillatory transenantiomerization of $L-\alpha$-phenylalanine is given by Eq. (2), although this equation does not reflect the elementary steps involved. Below, we present an outline of a plausible skeleton mechanism in the form of a set of elementary 


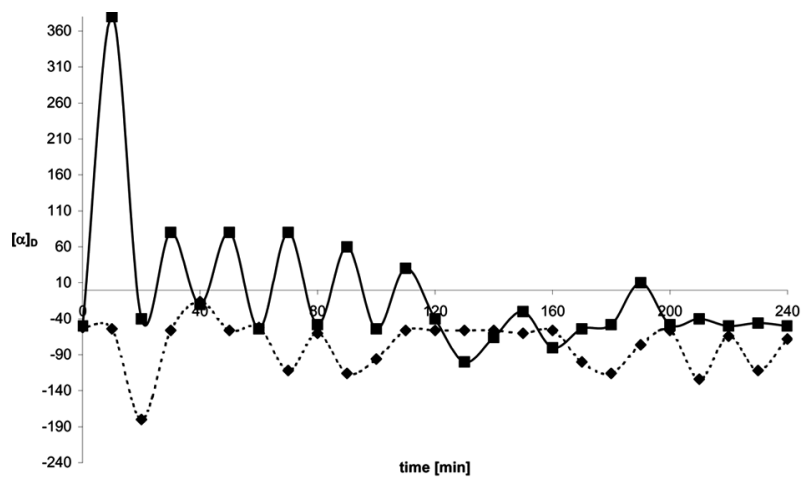

(a)

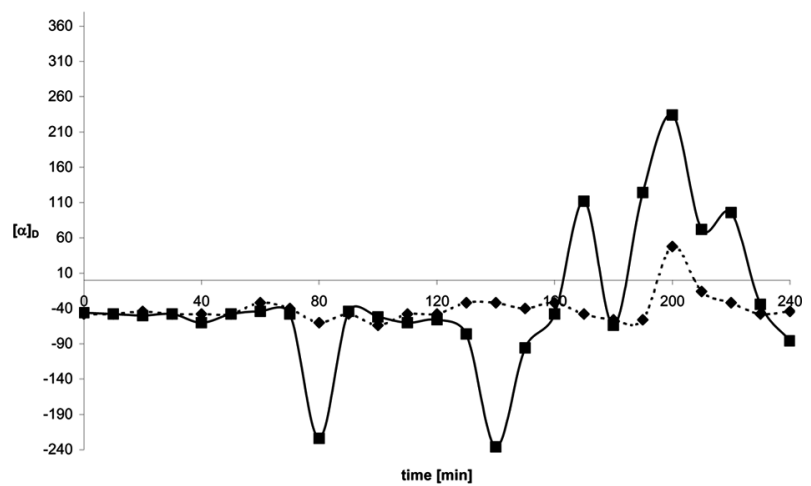

(b)

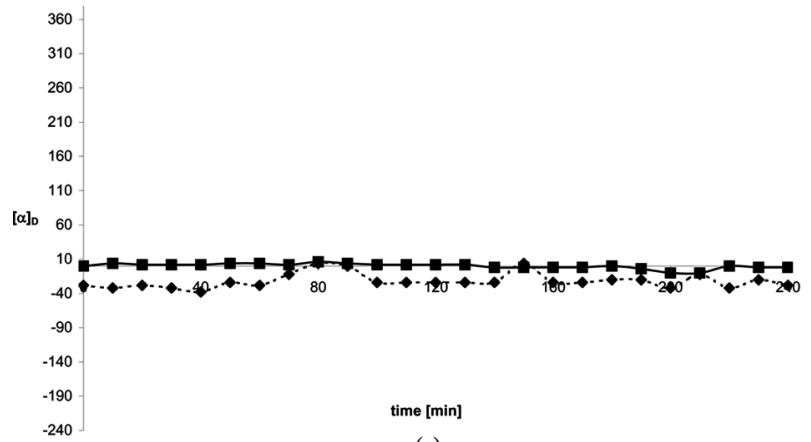

(c)

Figure 6. Comparison of the oscillations of the specific rotation value $\left([\alpha]_{D}\right)$ for $L$ - $\alpha$-phenylalanine dissolved and stored for $240 \mathrm{~min}$ at $9 \pm 2{ }^{\circ} \mathrm{C}$ in (a) ethanol + water $(7: 3, \mathrm{v} / \mathrm{v}),(\mathrm{b})$ ethanol + basic buffer, $\mathrm{pH}=9(7: 3, \mathrm{v} / \mathrm{v})$, and (c) ethanol + glacial acetic acid $(7: 3, \mathrm{v} / \mathrm{v})$ with and without ultrasonication (solid line: without ultrasonication; dashed line: with ultrasonication). 
chemical reactions that we posit to play a role in the amino acid transenantiomerization.

Enolization

$$
\begin{aligned}
& L+B \leftrightarrow E+B ; k_{e 1}, k_{e-1} \\
& D+B \leftrightarrow E+B ; k_{e 1}, k_{e-1}
\end{aligned}
$$

Dimerization

$$
\begin{gathered}
L+L \leftrightarrow L_{2} ; k_{d 1}, k_{d-1} \\
D+D \leftrightarrow D_{2} ; k_{d 1}, k_{d-1} \\
L+D \leftrightarrow L D ; k_{d 2}, k_{d-2} \quad\left(\text { where } K_{d 2}<K_{d 1}\right)
\end{gathered}
$$

Catalysis

$$
\begin{gathered}
E+L_{2} \rightarrow 3 L ; k_{c 1} \\
E+D_{2} \rightarrow 3 D ; k_{c 1} \\
E+L D \rightarrow 2 L+D ; k_{c 2} \\
E+L D \rightarrow L+2 D ; k_{c 2}
\end{gathered}
$$

where $L$ and $D$ are the $L$ - and $D$-enantiomers, respectively, $E$ is the keto-enol, $B$ is base, $L_{2}$ and $D_{2}$ are the homodimers (held together by hydrogen bonds) of $L$ and $D$, respectively, LD is the H-bonded heterodimer of $L$ and $D$, the $k$ 's are the respective reaction rates, and the $K$ 's are the corresponding equilibrium constants.

Based upon the results of our experiments and the known properties of keto-enol tautomerism, we take the first step of our mechanism (i.e., enolization) to be the base-catalyzed formation of the keto-enol derived from either enantiomer $L$ or enantiomer $D$ (the reversible reactions given by Eqs. (6) and (7)).

As 2-APAs are carboxylic acids, they interact through hydrogen bonds. When dissolved in low polarity solvents, they tend to form cyclic dimers, while in polar solvents, they can also form mixed associative multimers. The dimerization constitutes step two of the mechanism (parallel to step one), labeled as dimerization and described by the reversible Eqs. (8)-(10).

Step three - catalysis - is crucial for the interconversion of the chiral 2-APA, and it is described by the four irreversible Eqs. (11)-(14). Eqs. (11) and (12) express the critical assumption that each homodimer ( $L_{2}$ or $D_{2}$, respectively) catalyzes generation of, i.e., acts as a template for, the corresponding enantiomer from the keto-enol (whose formation is, in turn, catalyzed by the base). In the case of the heterodimer LD, 
we assume that it catalyzes formation of the enantiomers $L$ and $D$ with equal probability, in a non-stereospecific way (Eqs. (13) and (14)).

In order to properly analyze the kinetic equations corresponding to the stoichiometric Eqs. (6)-(14), one ought to know at least the orders of magnitude of each of the rate constants, which are, in fact, unavailable and extremely difficult, if not impossible, to measure. Rather than trying to fit all of the parameters of our mechanism quantitatively, we decided to focus on the qualitative behavior of the catalysis portion of our mechanism and to represent this aspect by an even simpler mechanism, the Templator. ${ }^{[1,12]}$

The Templator model attempts to capture the essential elements of the dynamic behavior of self-replicating systems ${ }^{[13,14]}$ in three elementary steps:

$$
\begin{gathered}
A_{0} \rightarrow A ; \quad \text { rate } k_{0} a_{0} \\
2 A+B \rightarrow 2 B ; \quad \text { rate } k_{1} a^{2} b \\
B \rightarrow P ; \quad \text { rate } k_{2} /(K+b)
\end{gathered}
$$

where $a=[A], b=[B]$, etc. The monomer A flows continuously into the system (15) and undergoes dimerization to form the dimer $B$, which acts as a template for its own formation (16). $B$ is transformed via a catalytic reaction (17) to the inert product $P$.

Reactions (15)-(17) constitute a rather crude caricature of our system, but they contain the essential aspect of multimerpromoted transformation and, with only two species, $A$ and $B$, whose concentrations need to be followed, they are simple enough to allow simulation and analysis of their dynamics, even when coupled to a second Templator. Let A and B represent the monomeric and dimeric forms, respectively, of the $L$-enantiomer. We introduce a second set of reactions, analogous to reactions (15)-(17) involving the corresponding $D$-species, which we designate as $A^{\prime}$ and $B^{\prime}$. We also add the reversible interconversion of the monomeric species via keto-enol tautomerization:

$$
A \rightarrow A^{\prime} ; \quad \text { rate } k_{r}\left(a-a^{\prime}\right)
$$

The rate equations for our coupled system are:

$$
\begin{gathered}
d a / d t=k_{0} a_{0}-2 k_{1} a^{2} b+k_{r}\left(a^{\prime}-a\right) \\
d b / d t=k_{1} a^{2} b-\frac{k_{2} b}{b+K} \\
d a^{\prime} / d t=k_{0} a_{0}^{\prime}-2 k_{1} a^{\prime 2} b^{\prime}-k_{r}\left(a^{\prime}-a\right) \\
d b^{\prime} / d t=k_{1} a^{\prime 2} b^{\prime}-\frac{k_{2} b^{\prime}}{b^{\prime}+K}
\end{gathered}
$$


Since we are interested primarily in the qualitative behavior of our model, we chose the simple set of parameter values $k_{0} a_{0}=1, k_{1}=1$, $k_{2}=1, k_{e}=0.1, K=0.1$ and investigated the behavior of the system at various values of $k_{0} a_{0}^{\prime}$. Note that, by rescaling the variables or, equivalently, choosing appropriate units for the time and concentrations, it is possible to assign any desired value to a particular parameter without changing the qualitative behavior of the system. We numerically

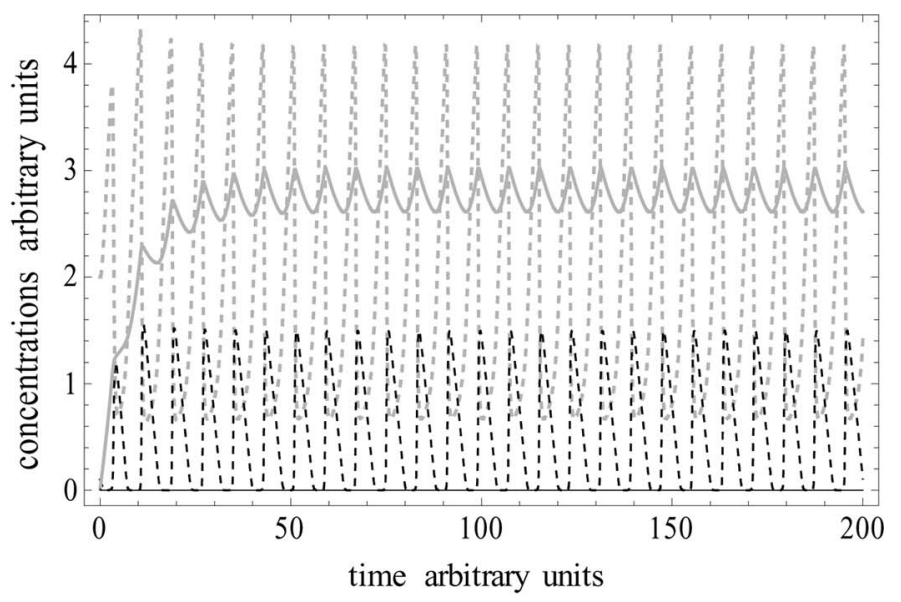

(a)

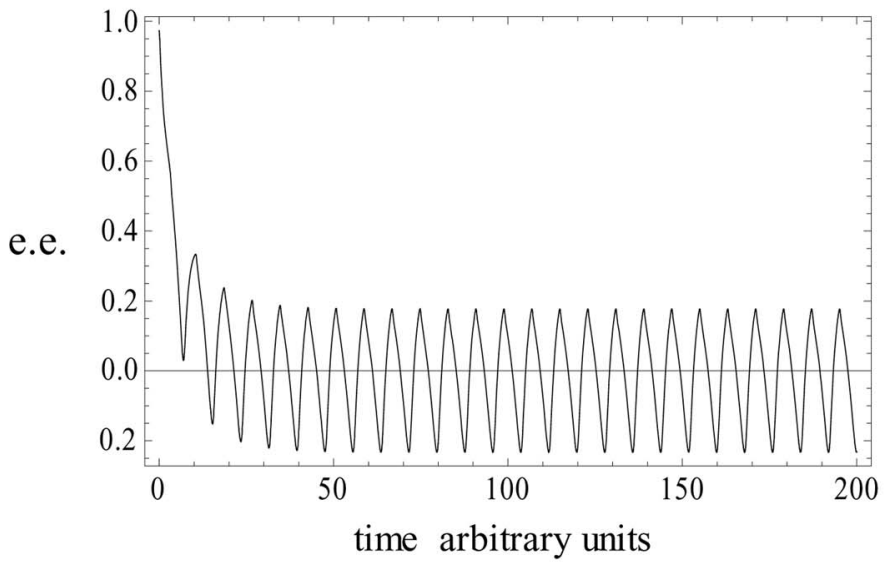

(b)

Figure 7. Simulations of model (19)-(22) with $k_{0} a_{0}^{\prime}=(a, b) 0.1$, (c,d) 0.2. Initial conditions $a_{0}=2, b_{0}=0.1, a_{0}^{\prime}=0.01, b_{0}^{\prime}=0.01$. In (a) and (c) gray dashed line and gray solid line show $a$ and $b$, respectively; black dashed line and black solid line show $a^{\prime}$ and $b^{\prime}$, respectively. 
integrated Eqs. (19)-(21) using the Matlab software package and obtained oscillatory behavior over a wide range of parameters. We define the enantiomeric excess (e.e.) as

$$
\text { e.e. }=\left(a+2 b-a^{\prime}-2 b^{\prime}\right) /\left(a+2 b+a^{\prime}+2 b^{\prime}\right)
$$

i.e., as the difference between total $L$-monomer and total $D$-monomer concentrations divided by the total monomer concentration.

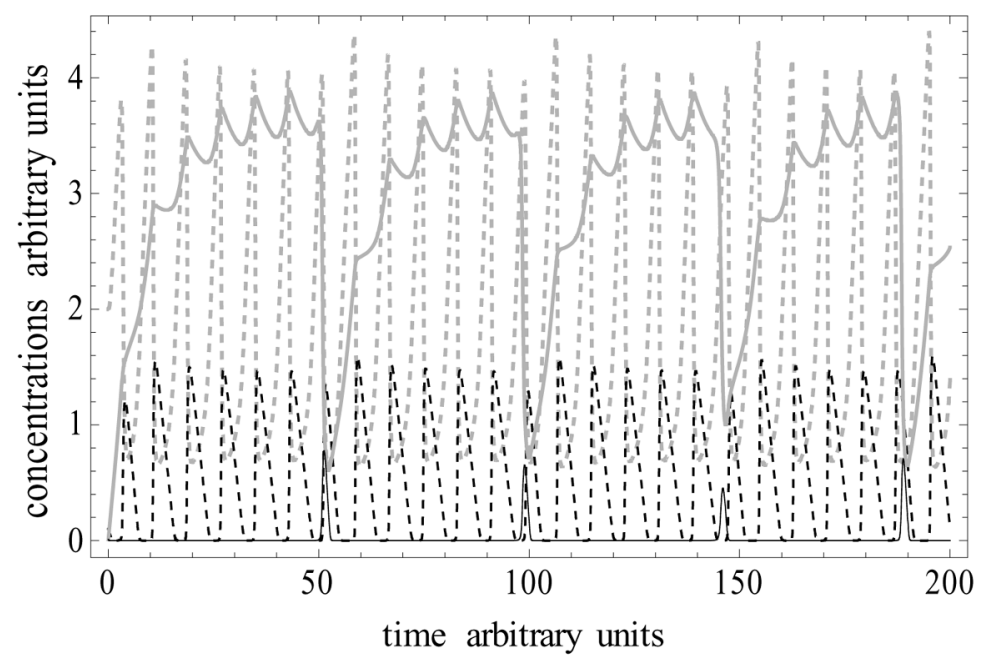

(c)

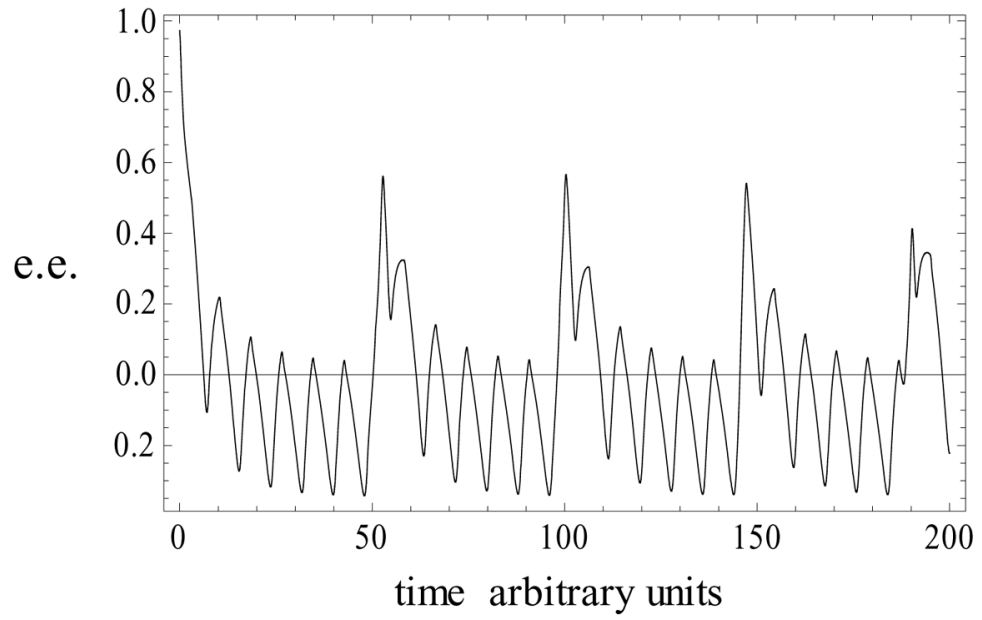

(d)

Figure 7. Continued 
In Figure 7, we show the results obtained for two values of $k_{0} a_{0}^{\prime}$. In effect, the ratio $a_{0}^{\prime} / a_{0}$ gives the fraction of impurity in the nominally optically pure starting material. In both cases, the behavior becomes periodic after a brief initial transient, and the sign of e.e. oscillates from positive to negative. For the higher level of impurity, the behavior is quite complex, with several maxima and minima per cycle, consistent with some of the irregular behavior seen in studies of the profens. ${ }^{[1-3]}$ For other choices of the kinetic parameters, the system either behaves monotonically or oscillates in such a fashion that e.e. never changes sign.

We also explored whether our coupled Templator model can give rise to spatial patterning. To do this, we added diffusion terms to Eqs. (19)-(22) for a one-dimensional (line) system of length 10. We took the diffusion coefficients of $A$ and $A^{\prime}$ to be equal and the diffusion coefficients of the dimeric species to be significantly smaller than those of the monomers, which is necessary, though not sufficient, for Turing pattern formation. ${ }^{[15]}$ Starting from the parameters and homogeneous initial conditions corresponding to Fig. 7(c) and 7(d), we added a small random perturbation to the concentration of $\mathrm{A}$ and followed its evolution in time and space. The results are shown as space-time plots in Fig. 8. Looking parallel to the horizontal axis of Figs. 8(a) and 8(b), we observe a well-defined spatial structure for the $L$-form, with regions of high $a$ and low $b$ alternating with regions of low $a$ and high $b$. Along the vertical axis, we observe a temporal periodicity as well. Interestingly, $a^{\prime}$ and $b^{\prime}$ [Figs. 8(c) and (d)] are almost homogeneously distributed in space, though they oscillate periodically in time. The e.e. plot in Fig. 8(e) reflects, primarily, the structure of the $B$ concentration profile.

Summing up, our explanation of the oscillatory in vitro chiral inversion of $L-\alpha$-phenylalanine is grounded in the molecular structure of the compound, in particular its ability to intermolecularly interact through hydrogen bonds to form supramolecular structures of H-bonded cyclic dimers and/or H-bonded linear multimers. These structural features are essential for the enantioselective templating process that lies at the heart of our mechanism.

Qualitative agreement between our experimental results and theoretical simulations can best be seen from a comparison of the oscillatory nature of the plots shown in Figs. 2 and 6 (experiment) with those given in Fig. 7 (theory). The oscillatory plots from Figs. 2, 6 , and 7 illustrate, respectively, the time behavior of the experimental retention parameter $\left(R_{\mathrm{F}}\right)$, the polarimetrically measured specific rotation $\left([\alpha]_{D}\right)$, and the simulated concentration changes of the two antimers $\left(a, a^{\prime}, b, b^{\prime}\right)$. Qualitative agreement is also found between the stripes in the photograph in Fig. 5 (experiment) and the simulated patterns in Fig. 8 (theory), though the latter only demonstrates spatial concentration anisotropy of the two antimers in one spatial dimension. 


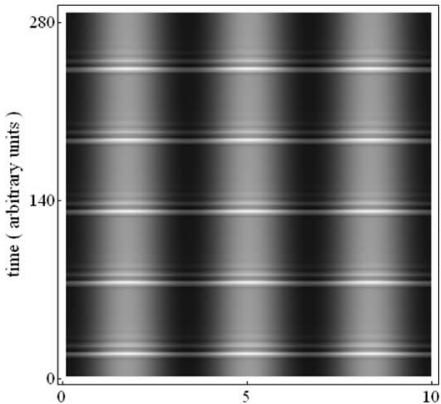

space ( arbitrary units )

(a)

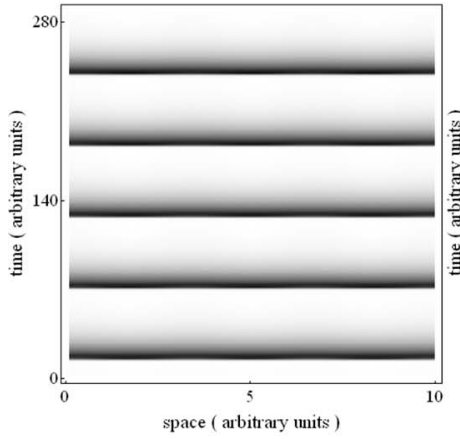

(c)

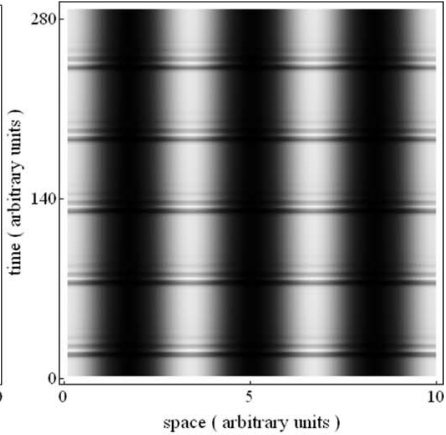

(b)

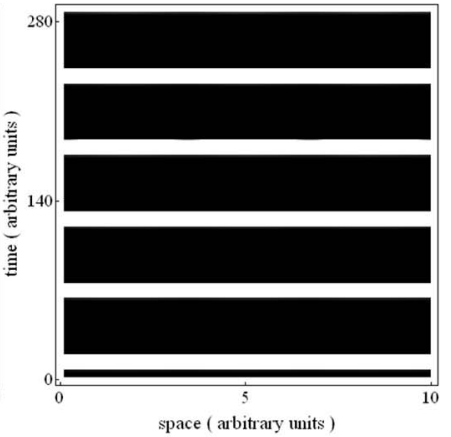

(d)

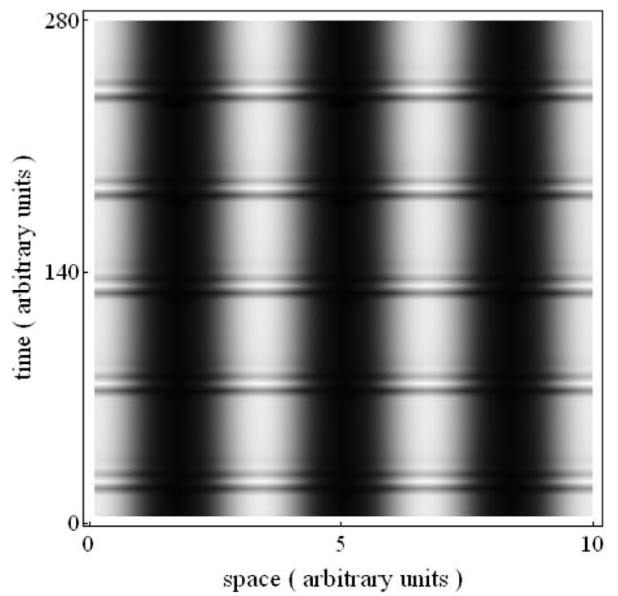

(e)

Figure 8. Space-time plot of simulated concentrations with model (19)-(22) augmented with diffusion of all species. Diffusion coefficients $D_{A}=D_{A}^{\prime}=1$, $D_{B}=D_{B^{\prime}}=0.125$. Initial conditions and parameters as in Fig. 7c,d. White corresponds to high concentrations, black to low concentrations. (a) $a$, (b) $b$, (c) $a^{\prime}$, (d) $b^{\prime}$, (e) e.e. 
Our model explanation of the oscillatory in vitro chiral inversion of $L-\alpha$-phenylalanine is quite general and not restricted to this compound alone. It is not limited to amino acids with a chemical structure that can formally be derived from propionic acid. It can be applied to profen drugs as well, though the limits of its applicability have not yet been explored in detail. One may also be able to apply this model to chiral carboxylic acids formally derived from butyric acid, or from even higher aliphatic acids (i.e., to such amino acids as valine, leucine, and threonine, and to such drugs as bufens). On the other hand, with increasing aliphatic chain length, the ability of carboxylic acids to form intermolecular hydrogen bonds will gradually diminish (due to the shielding effect of the flexible aliphatic chain) and, accordingly, their ability to undergo the oscillatory in vitro chiral inversion should also decrease. Of course, the generality of our model also implies that, in the absence of detailed data on the kinetic parameters for a particular chiral species, we can only derive qualitative rather than quantitative conclusions from our simluations.

In future studies, we will focus on the search for new chiral compounds and compound classes that show an analogous ability to undergo oscillatory chiral inversion and on studying the relationship between their molecular structure and the kinetics of their oscillations. Our experience suggests that thin-layer chromatography is a particularly promising tool for this kind of investigation, and we will continue to employ this technique in our investigations.

\section{ACKNOWLEDGMENT}

The authors thank Merck KGaA (Darmstadt, Germany) for supplying the TLC plates used in our experiments. This work was supported in part by the U.S. National Science Foundation (grant CHE-0615507).

\section{REFERENCES}

1. Sajewicz, M.; Piętka, R.; Pieniak, A.; Kowalska, T. Application of thin-layer chromatography (TLC) to investigating oscillatory instability of the selected profen enantiomers. Acta Chromatogr. 2005, 15, 131-149.

2. Sajewicz, M.; Piętka, R.; Pieniak, A.; Kowalska, T. Application of thinlayer chromatography (TLC) to investigate oscillatory instability of the selected profen enantiomers in dichloromethane. J. Chromatogr. Sci. 2005, 43, 542-548.

3. Sajewicz, M.; Piętka, R.; Pieniak, A.; Kowalska, T. Application of thin-layer chromatography to the investigation of oscillatory instability of selected profen enantiomers in physiological salt. J. Liq. Chromatogr. Rel. Technol. 2006, 29, 2059-2069. 
4. Sajewicz, M.; Piętka, R.; Drabik, G.; Kowalska, T. On the mechanism of oscillatory changes of the retardation factor $\left(R_{\mathrm{F}}\right)$ and the specific rotation $[\alpha]_{D}$ with selected solutions of $S$-(+)-naproxen. J. Liq. Chromatogr. Rel. Technol. 2006, 29, 2071-2082.

5. Virtual catalog of ScienceLab.com (http://sciencelab.com/)

6. Bhushan, R.; Reddy, G.P.; Joshi, S. TLC resolution of DL amino acids on impregnated silica gel plates. J. Planar Chromatogr. 1994, 7, 126-128.

7. Gray, P.; Scott, S.K. Chemical Oscillations and Instabilities. Non-Linear Chemical Kinetics. Clarendon Press, Oxford, 1994.

8. Sajewicz, M.; Piętka, R.; Kus, P.; Kowalska, T. On the gelation of profens as a property causing their oscillatory transenantiomerization. Acta Chromatogr. 2006, 16, 181-191.

9. Sajewicz, M.; Gontarska, M.; Kronenbach, D.; Wojtal, Ł.; Grygierczyk, G.; Kowalska, T. A study on the oscillatory in vitro transenantiomerization and enantioseparation of the flurbiprofen antimers by means of thin layer chromatography (TLC). Acta Chromatogr. 2007, 18, 227-238.

10. Sajewicz, M.; Epstein, I.R.; Kowalska, T. Proceedings of the 7th Balaton Symposium on High Performance Separation Methods (In Memoriam Szabolcs Nyiredy), Sept. 5th-7th, Siofok, Hungary, 2007.

11. Sajewicz, M.; Gontarska, M.; Wojtal, Ł.; Epstein, I.R.; Kowalska, T. Proceedings of the 7th Balaton Symposium on High Performance Separation Methods (In Memoriam Szabolcs Nyiredy), Sept. 5th-7th, Siofok, Hungary, 2007.

12. Peacock-Lopez, E.; Radov, D.B.; Flesner, C.S. Mixed-mode oscillations in a self-replicating dimerization mechanism. Biophys. Chem. 1997, 65, 171-178.

13. Tsai, L.L.; Hutchinson, G.R.; Peacock-Lopez, E. Turing patterns in a selfreplicating mechanism with a self-complementary template. J. Chem. Phys. 2000, 113, 2003-2006.

14. Tjivikua, T.; Ballester, P.; Rebek, J. A self-replicating system. J. Am. Chem. Soc. 1990, 112, 1249-1250.

15. Turing, A.M. The chemical basis of morphogenesis. Phil. Trans. Roy. Soc. London, 1952, B237, 37-72.

Received November 28, 2007

Accepted December 14, 2007

Manuscript 6311B 